\title{
Negative contrast effect as a function of magnitude of reward decrement'
}

\author{
VINCENT DI LOLLO, UNIVERSITY OF WESTERN AUSTRALIA \\ VICTOR BEEZ, INDIANA UNIVERSITY
}

Five groups of 12 rats each were given 20 daily training trials in a straight runway and were rewarded with either $1,2,4,8$ or $1620 \mathrm{mg}$ food pellets, respectively. All groups were then shifted to 1 pellet reward for 14 additional trials. Pcrformance during Training was an increasing monotonic function of the amount of reward. The magnitude of the negative contrast effect obtained in post-shift performance was directly related to the magnitude of reward decrement.

Following a sudden decrement in the amount of reward, the performance of a group of animals typically decreases below that of a control group maintained at the lower amount throughout. Although the occurrence of this phenomenon, termed negative contrast effect, has been repeatedly demonstrated, little work has been done towards a description of its relation to relevant variables connected with the shift in reward.

The effect of magnitude of shift in reward was first studied by Crespi (1942) with inconclusive results. In a more recent study (Gonzales, Gleitman, \& Bitterman, 1962) a positive relationship was obtained between magnitude of reward decrement and negative contrast effect; these investigators, however, used only two levels of reward decrement and thus did not obtain an indication of the function relating the two variables. The study here reported investigates the magnitude of the negative contrast effect as a function of the magnitude of decrement in reward over a range of five levels of the independent variable.

Method

Apparatus. The apparatus consisted of a straight runway $86 \mathrm{in.} \mathrm{long,} 3 \mathrm{in}$. wide and $4 \mathrm{in}$. high; the length included a 12 in. start boxand a 14 in. goal box separated from the alley by clear plastic guillotine doors. A softdrink bottle top was attached to the floor of the far end of the goal box and acted as a food cup. The apparatus was painted flat black and was covered with clear Plexiglas. Running times were measured by a .01 sec. Standard Electric timer between a photo beam 12 in. past the start door and a second beam located 36 in. from the first.

Subjects and procedure. Sixty male albino rats, 90 to 100 days old, were allocated randomly to five groups of 12 Ss each. Fourteen days before the beginning of training the Ss were placed on a feeding schedule with food available for $1 \mathrm{hr}$. each day and water available at all times. During Training each $S$ ran one trial per day for 20 days. The reward consisted of either $1,2,4,8$, or $1620 \mathrm{mg}$ Noyes food pellets for each of the five groups. respectively. On the last day of Training, and for 14 subsequent daily trials, the reward was shifted to one pellet for all groups (Shift stage).

\section{Results and Discussion}

The running time of each $S$ on each trial was transformed logarithmically and then averaged for each group over the last five trials of Training and Shift, respectively, to give the measure of performance shown in Fig. 1. The one-tailed probability of obtaining an ordered replication of the five amounts of reward during Training or Shift is $1 / 5 !(p<.01)$. Clearly, the magnitude of the negative contrast effect is a monotonic function of the magnitude of reward decrement under the present experimental conditions.

During Training, performance was an approximately linear function of the $\log$ amount of reward (Fig. 1). This result is in agreement with earlier work (e.g., Guttman, 1953) and is consonant with predictions from the incentive motivation theory proposed by Bevan \& Adamson (1960).

As shown in Fig. 2, changes in performance during Shift were gradual and, apparently, not quite complete at the end of the experiment. A similarly slow onset of the negative contrast effect was obtained in some earlier studies (Meyer, 1951; Spence, 1956; Collier \& Marx, 1959), while rapid shifts were obtained in others (Di Lollo, 1965; Vogel, Mikulka, \& Spear, 1965). Some of the variables possibly affecting the rapidity of the shifts have recently been discussed by Ison \& Rosen (1965): the issue, however, is complex and has not been resolved.

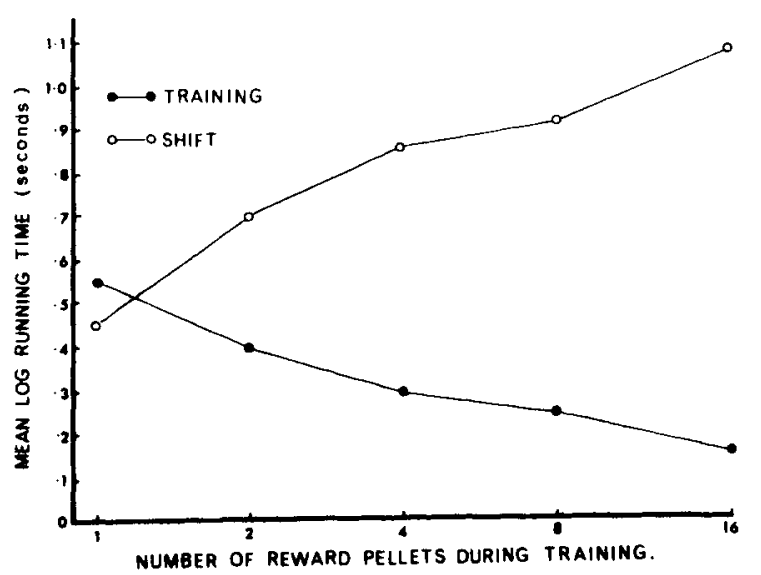

Fig. 1. Mean log running time for each Group over the last five trials of Training and Shift, respectively 


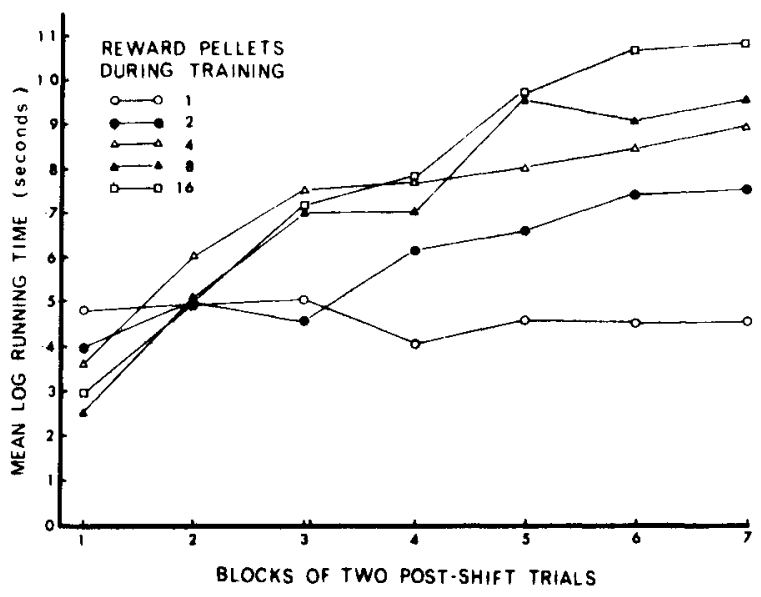

Fig. 2. Mean log running time for each Group during Shift.

Although both perceptual-motivational theory (Bevan \& Adamson, 1960) and response-motivational theory (Crespi, 1944; Spence, 1956) can account for the graded magnitude of the contrast effect, the gradual onset of the phenomenon is not in agreement with predictions from either formulation. In its present form, each theory predicts converging, rather than diverging, post-shift performance curves. The gradual changes in performance suggest that, under some yet unspecified conditions, the development of the negative contrast effect is governed by learning, as distinct from motivational, variables. A statement of such variables should be encompassed within any theory aiming at a complete account of the phenomenon.

\section{References}

Bevan, W., \& Adamson, $\mathbf{R}$. Reinforcers and reinforcement: their relation to maze performance. J. exp. Psychol., 1960, 59, 226232.

Collier, G., \& Marx, M. H. Changes in performance as a function of shifts in the magnitude of reinforcement. J. exp. Psychol., $1959,57,305-309$.

Crespi, I. P. Quantitative variations of incentive and performance in the white rat. Amer. J. Psychol., 1942, -55, 467-517.

Crespi, L. P. Amount of reinforcement and level of performance, Psychol. Rev., 1944, 51, 341-357.

Di Lollo, V. Runway performance in relation to runway-goal-box similarity and changes in incentive amount. J. comp. physiol. Psychol, , 1964, 58, 327-329.

Gonzales, R. C., Gleitman, H., \& Bitterman, M. E. Some observations on the depression effect. J. comp. physiol. Psychol., 1962, 55, 578-581.

Guttman, N. Operant conditioning, extinction, and periodic reinforcement in relation to concentration of sucrose used as a reinforcing agent. J. exp. Psychol., 1953, 46, 213-224.

Ison, J. R., \& Rosen, A. J. On changes in incentive: some new findings and theoretical speculations. Paper read at meeting of Psychonomic Society, 1965.

Meyer, D. R. The effects of differential rewards on discrimination reversal learning by monkeys. J. exp. Psychol., 1951, 41, 268274.

Spence, K. W. Behavior theory and conditioning. New Haven: Yale University Press, 1956.

Vogel, J. R., Mikulka, P. J., \& Spear, N. E. Effects of interpolated extinction and level of training on the "depression effect". Paper read at meeting of the Eastern Psychological Association, 1965.

\section{Note}

1. The experimental work reported in this paper was conducted at Indiana University and was supported in part by Grant GE 2904 from the National Science Foundation. Analyses of the data and preparation of this report were supported by the Air Force Office of Scientific Research, Office of Aerospace Research, United States Air Force, under AFOSR Grant Nr AF - AFOSR - 968 - 65. 